\title{
On non-local reaction-diffusion system in a bounded domain
}

Mauricio Bogoya* (1)

\section{*Correspondence:}

mbogoyal@unal.edu.co

Departamento de Matemáticas, Universidad Nacional de Colombia, Bogotá, Colombia

\section{照 Springer}

$$
\begin{aligned}
& \text { Abstract } \\
& \text { We study the non-local reaction-diffusion system with Neumann boundary } \\
& \text { conditions } \\
& \qquad \begin{aligned}
u_{t}(x, t) & =\int_{\Omega} J(x-y)(u(y, t)-u(x, t)) d y+v^{p}(x, t), \quad(x, t) \in \Omega \times(0, T), \\
v_{t}(x, t) & =\int_{\Omega} J(x-y)(v(y, t)-v(x, t)) d y+u^{q}(x, t), \quad(x, t) \in \Omega \times(0, T), \\
u(x, 0) & =u_{0}(x), \quad v(x, 0)=v_{0}(x), \quad x \in \Omega,
\end{aligned}
\end{aligned}
$$

where $p, q>0, u_{0}(x), v_{0}(x) \in C(\bar{\Omega})$ are nonnegative and nontrivial functions, $\Omega \in \mathbb{R}^{N}$ a bounded connected and smooth domain. We determine the existence and uniqueness of the solution. The blow-up phenomenon is considered and the blow-up rates are obtained.

MSC: 35B44; 35K40; 35K57

Keywords: Non-local diffusion; System equations; Neumann boundary conditions; Blow-up

\section{Introduction}

The following coupled parabolic system with Neumann boundary conditions:

$$
\begin{aligned}
& u_{t}=\Delta u+v^{p}, \quad(x, t) \in \Omega \times(0, T), \\
& v_{t}=\Delta v+u^{q}, \quad(x, t) \in \Omega \times(0, T), \\
& \frac{\partial u}{\partial \eta}(x, t)=0, \quad \frac{\partial u}{\partial \eta}(x, t)=0, \quad(x, t) \in \partial \Omega \times(0, T), \\
& u(x, 0)=u_{0}(x), \quad v(x, 0)=v_{0}(x), \quad x \in \Omega,
\end{aligned}
$$

where $p, q>0$ and $\Omega \subset \mathbb{R}^{N}(N \geq 1)$ is a bounded domain with smooth boundary with $\eta$ the outer normal, has been studied recently. In particular, the existence and uniqueness of the solution and the blow-up phenomena have been analyzed in Refs. [1-8].

Pao [5], analyzes the system (1.1) in the case the reaction terms $f$ and $g$ are quasimonotone nondecreasing functions. The existence and uniqueness of the solution is studied using the technique of approximation by upper and lower solutions. Moreover, have

(c) The Author(s) 2018. This article is distributed under the terms of the Creative Commons Attribution 4.0 International License (http://creativecommons.org/licenses/by/4.0/), which permits unrestricted use, distribution, and reproduction in any medium, provided you give appropriate credit to the original author(s) and the source, provide a link to the Creative Commons license, and indicate if changes were made. 
been showed, for any nonnegative initial conditions $u_{0} \neq 0, v_{0} \neq 0$ and $p q>1$ the solution $(u, v)$ of (1.1) blows up at a finite time $T$.

In the case of a single equation

$$
\begin{aligned}
& u_{t}=\Delta u+u^{p}, \quad(x, t) \in \Omega \times(0, T), \\
& \frac{\partial u}{\partial \eta}(x, t)=0, \quad(x, t) \in \partial \Omega \times(0, T) ; \quad u(x, 0)=u_{0}(x), \quad x \in \Omega,
\end{aligned}
$$

Friedman et al. [9] have found for $p>1$ that the solution blows up in a finite time, moreover, they gave the blow-up rates [10-12].

Shahmurov [13] studied the Dirichlet and Neumann problems for a special Helmholtz equation on an annulus. The aim of that work was to measure smoothness of solutions for the boundary datum in Besov spaces. The author used the operators theory for handle this problem. The advantage of that technique is that we can consider equations with vectorvalued.

We said the solution $(u, v)$ blows up in finite time if only if exists a finite time $T>0$ such that

$$
\lim _{t>T} \sup \left(\|u(x, t)\|_{L^{\infty}(\Omega)}+\|v(x, t)\|_{L^{\infty}(\Omega)}\right)=\infty
$$

If $T=\infty$ the solution $(u, v)$ is global, i.e. the solution exists for all $t \geq 0$.

Equations of the form

$$
u_{t}(x, t)=J * u-u(x, t)=\int_{\mathbb{R}^{n}} J(x-y) u(y, t) d y-u(x, t)
$$

and variations of it, have been widely used in the last decade to model diffusion processes, see for instance Refs. [14-17]. As stated in [17] if $J: \mathbb{R}^{n} \rightarrow \mathbb{R}$ be a nonnegative, smooth, symmetric $(J(-z)=J(z))$ and strictly decreasing function, with $\int_{\mathbb{R}^{n}} J(x) d x=1, J$ supported in the unit ball and if $u(x, t)$ is thought of as a density at the point $x$ at time $t$, and if $J(x-y)$ is thought of as the probability distribution of jumping from location $y$ to location $x$, then $(J * u)(x, t)$ is the rate at which individuals are arriving to position $x$ from all other places and $-u(x, t)=-\int_{\mathbb{R}^{n}} J(y-x) u(x, t) d y$ is the rate at which they are leaving location $x$ to travel to all other sites. This consideration, in the absence of external sources, leads immediately to the fact that the density $u$ satisfies Eq. (1.3). This equation is called a non-local diffusion equation since the diffusion of the density $u$ at a point $x$ and time $t$ does not only depend on $u(x, t)$, but also on all the values of $u$ in a neighborhood of $x$ through the convolution term $J * u$. This equation shares many properties with the classical heat equation $u_{t}=\Delta u$ : bounded stationary solutions are constant, a maximum principle holds for both of them, and even if $J$ is compactly supported, perturbations propagate with infinite speed; see [17]. However, there is no regularizing effect in general [18].

Chasseigne et al. [18] studied the Neumann boundary conditions problem

$$
\begin{aligned}
& u_{t}(x, t)=\int_{\Omega} J(x-y)(u(y, t)-u(x, t)) d y, \quad x \in \Omega, t>0, \\
& u(x, 0)=u_{0}(x), \quad x \in \Omega,
\end{aligned}
$$


where $u_{0} \in L^{1}(\Omega)$ is a nonnegative function and $\Omega \subset \mathbb{R}^{N}$ a bounded connected and smooth domain. Since the integration is in $\Omega$, they impose the requirement that the diffusion takes place only in $\Omega$. The individuals may not enter nor leave $\Omega$. This is the analogous of what is called homogeneous Neumann boundary conditions in the literature. One analyzed the existence and uniqueness of solutions for (1.4) and found an exponential convergence to the mean value of the initial condition.

Pérez-Llanos and Rossi [19] studied the equation with a reaction term,

$$
\begin{aligned}
& u_{t}(x, t)=\int_{\Omega} J(x-y)(u(y, t)-u(x, t)) d y+u^{p}(x, t), \quad(x, t) \in \Omega \times(0, T), \\
& u(x, 0)=u_{0}(x), \quad x \in \Omega
\end{aligned}
$$

for $p>0, \Omega \subset \mathbb{R}^{N}$ a bounded connected and smooth domain and $u_{0} \in C(\bar{\Omega})$ a nonnegative function. They proved the nonnegative and nontrivial solutions blow up in finite time if and only if $p>1$. Moreover, they find the blow-up rate is the same as the one that holds for the ODE $u_{t}=u^{p}$, that is, $\lim _{t \rightarrow T^{-}}(T-t)^{1 /(p-1)}\|u(\cdot, t)\|_{\infty}=(1 /(p-1))^{1 /(p-1)}$.

The problem (1.5) shares properties with the corresponding local diffusion problem (1.2). García Melián and Quiróz [20] studied Eq. (1.5) in the case $\Omega=\mathbb{R}^{N}$.

Our main objective in this paper is the study of the following non-local reactiondiffusion system with Neumann boundary conditions:

$$
\begin{aligned}
& u_{t}(x, t)=\int_{\Omega} J(x-y)(u(y, t)-u(x, t)) d y+v^{p}(x, t), \quad(x, t) \in \Omega \times(0, T), \\
& v_{t}(x, t)=\int_{\Omega} J(x-y)(v(y, t)-v(x, t)) d y+u^{q}(x, t), \quad(x, t) \in \Omega \times(0, T), \\
& u(x, 0)=u_{0}(x), \quad v(x, 0)=v_{0}(x), \quad x \in \Omega,
\end{aligned}
$$

with $p, q>0, u_{0}(x), v_{0}(x) \in C(\bar{\Omega})$ nonnegative and nontrivial functions and $\Omega \subset \mathbb{R}^{N}(N \geq 1)$ a bounded connected and smooth domain. We are imposing the requirement that diffusion takes place only in $\Omega$, no individual may enter or leave the domain, that is, we have Neumann boundary conditions.

First, we analyze existence of nonnegative solutions $(u, v)$ of (1.6). We show the solution $(u, v)$ is unique if $p q \geq 1$ or if at least one of the initials conditions is not zero for $p q<1$. Next, we analyze the globally existence. We show that if $p q>1$ and $u_{0}, v_{0}$ are nonnegative and nontrivial functions the solution $(u, v)$ blows up in finite time $T$, and if $p q \leq 1$ the solution $(u, v)$ exists globally. Finally, we analyze the blow-up rate. The results obtained allow us to conclude the system (1.6) shares important properties with the corresponding local diffusion coupled parabolic system with Neumann boundary conditions (1.1).

Non-local reaction-diffusion systems have applications concerned with thermal properties of visco-elastics and materials that have memory. Amann [21] presents a survey of the most common approaches to quasi-linear parabolic evolution equations, a discussion of their advantages and drawbacks, and a entirely new approach based on maximal Lp regularity.

The paper is organized as follows. In Sect. 2, we show the existence and uniqueness of solutions of (1.6). In Sect. 3, we study the global existence and the blow-up phenomenon. The blow-up rates are given. 


\section{Existence and uniqueness}

In this section, we show the existence and uniqueness of nonnegative solutions $(u, v)$ of (1.6) using the Banach fixed point theorem.

Let $t_{0}>0$ be fixed and

$$
X_{t_{0}}=C\left(\left[0, t_{0}\right]: C(\bar{\Omega}) \times C(\bar{\Omega})\right)=\left\{(u, v):\left[0, t_{0}\right] \rightarrow C(\bar{\Omega}) \times C(\bar{\Omega}):(u, v) \text { continuos }\right\},
$$

the Banach space with the norms

$$
\|(u, v)\|=\max _{0 \leq t \leq t_{0}}\|(u(\cdot, t), v(\cdot, t))\|_{I}, I=L^{\infty}(\bar{\Omega}) \times L^{\infty}(\bar{\Omega}),
$$

and

$$
\|(u(\cdot, t), v(\cdot, t))\|_{I}=\max _{x \in \bar{\Omega}}|u(x, t)|+\max _{x \in \bar{\Omega}}|v(x, t)| .
$$

Let $P_{t_{0}}=\left\{(u, v) \in X_{t_{0}}: u \geq 0, v \geq 0\right\}$ subspace of $X_{t_{0}}$ which is a closed subspace. We define the operator

$$
\psi: P_{t_{0}} \rightarrow P_{t_{0}}, \quad \text { as } \psi_{\left(u_{0}, v_{0}\right)}(u, v)=\left(T_{u_{0}}(u), S_{v_{0}}(v)\right)
$$

where

$$
\begin{aligned}
& T_{u_{0}}(u)(x, t)=\int_{0}^{t} \int_{\Omega} J(x-y)(u(y, s)-u(x, s)) d y d s+\int_{0}^{t}|v|^{p-1} v(x, s) d s+u_{0}(x), \\
& S_{v_{0}}(v)(x, t)=\int_{0}^{t} \int_{\Omega} J(x-y)(v(y, s)-v(x, s)) d y d s+\int_{0}^{t}|u|^{q-1} u(x, s) d s+v_{0}(x) .
\end{aligned}
$$

We denote $\|u\|_{\kappa}=\max _{0 \leq t \leq t_{0}}\|u(\cdot, t)\|_{L^{\infty}(\bar{\Omega})}$.

Lemma 2.1 Let $p, q \geq 1,\left(u_{0}, v_{0}\right),\left(w_{0}, z_{0}\right) \in C(\bar{\Omega}) \times C(\bar{\Omega})$ and $(u, v),(w, z) \in P_{t_{0}}$. Then there exists a positive constant $C=C\left(p, q,\|u\|_{\kappa},\|v\|_{\kappa},\|w\|_{\kappa},\|z\|_{\kappa}, \Omega, J\right)$ such that

$$
\left\|\mid \psi_{\left(u_{0}, v_{0}\right)}(u, v)-\psi_{\left(w_{0}, z_{0}\right)}(w, z)\right\|\left\|\leq C t_{0}\right\|(u, v)-(w, z)\|\|+\left\|\left(u_{0}, v_{0}\right)-\left(w_{0}, z_{0}\right)\right\|_{I} .
$$

Proof For any $(x, t) \in \bar{\Omega} \times\left[0, t_{0}\right]$ we have

$$
\begin{aligned}
& \left|T_{u_{0}}(u(x, t))-T_{w_{0}}(w(x, t))\right| \\
& \leq\left|u_{0}(x)-w_{0}(x)\right|+\int_{0}^{t} \int_{\Omega} J(x-y)|u(y, s)-w(y, s)| d y d s \\
& \quad+\int_{0}^{t} \int_{\Omega} J(x-y)|u(x, s)-w(x, s)| d y d s+\left.\int_{0}^{t}|| v\right|^{p-1} v(x, s)-|z|^{p-1} z(x, s) \mid d s \\
& \leq\left\|u_{0}-w_{0}\right\|_{L^{\infty}(\bar{\Omega})}+2 \int_{0}^{t}\|u(\cdot, s)-w(\cdot, s)\|_{L^{\infty}(\bar{\Omega})} d s \int_{\Omega} J(x-y) d y \\
& \quad+p \mu^{p-1} \int_{0}^{t}|v(x, s)-z(x, s)| d s \\
& \leq\left\|u_{0}-w_{0}\right\|_{L^{\infty}(\bar{\Omega})}+2 K t|\Omega|\|u-w\|_{\kappa}+t p \mu^{p-1}\|v-z\|_{\kappa},
\end{aligned}
$$

where $K=\|J\|_{\infty}$ and $\mu=\max \left\{\|v\|_{\kappa},\|z\|_{\kappa}\right\}$. 
Analogously we have

$$
\left|S_{v_{0}}(v(x, t))-S_{z_{0}}(z(x, t))\right| \leq\left\|v_{0}-z_{0}\right\|_{L^{\infty}(\bar{\Omega})}+2 K t|\Omega|\|v-z\|_{\kappa}+t q v^{q-1}\|u-w\|_{\kappa},
$$

where $v=\max \left\{\|u\|_{\kappa},\|w\|_{\kappa}\right\}$. Therefore, we obtain

$$
\begin{aligned}
& \|\| \psi_{\left(u_{0}, v_{0}\right)}(u, v)-\psi_{\left(w_{0}, z_{0}\right)}(w, z) \| \\
& \quad=\max _{0 \leq t \leq t_{0}}\left(\left\|T_{u_{0}} u-T_{w_{0}} w\right\|_{L^{\infty}(\bar{\Omega})}+\left\|S_{v_{0}} v-S_{z_{0}} z\right\|_{L^{\infty}(\bar{\Omega})}\right) \\
& \quad \leq\left\|\left(u_{0}, v_{0}\right)-\left(w_{0}, z_{0}\right)\right\|_{I}+\left(2 K|\Omega|+q v^{q-1}\right) t_{0}\|u-w\|+\left(2 K|\Omega|+p \mu^{p-1}\right) t_{0}\|v-z\| \\
& \quad=\left(2 K|\Omega|+k_{1}\right) t_{0}\|(u, v)-(w, z)\|+\left\|\left(u_{0}, v_{0}\right)-\left(w_{0}, z_{0}\right)\right\|_{I^{\prime}},
\end{aligned}
$$

where $k_{1}=\max \left\{p \mu^{p-1}, q v^{q-1}\right\}$. If $C=2 K|\Omega|+k_{1}$ we have

$$
\left\|\psi_{\left(u_{0}, v_{0}\right)}(u, v)-\psi_{\left(w_{0}, z_{0}\right)}(w, z)\right\|\left\|\leq t_{0}\right\|(u, v)-(w, z)\|\|+\left\|\left(u_{0}, v_{0}\right)-\left(w_{0}, z_{0}\right)\right\|_{I} .
$$

Remark 2.1 In [18], we find

$$
\begin{aligned}
& u_{t}(x, t)=\int_{\Omega} J(x-y)(u(y, t)-u(x, t)) d y, \quad x \in \Omega, t>0, \\
& u(x, 0)=h(x), \quad x \in \Omega
\end{aligned}
$$

where $h(x) \in L^{1}(\Omega)$, and $(x, t) \in \Omega \times\left(0, t_{0}\right)$ the solution is given by

$$
u(x, t)=h(x)+\int_{0}^{t} \int_{\Omega} J(x-y)(u(y, s)-u(x, s)) d y d s=: A_{u}(x, t) h
$$

Remark 2.2 Let $f(m)=m^{s}$, for $m>0$ and $s \in \mathbb{R}$. For $x \in \Omega$, we consider $\Omega^{+}=\{y \in \Omega$ : $u(y, t) \geq u(x, t)\}, \Omega^{-}=\{y \in \Omega: u(y, t)<u(x, t)\}$. Let $w_{\theta}(x, t)=\theta u(y, t)+(1-\theta) u(x, t)$, for $\theta \in(0,1)$. We have

$$
\begin{aligned}
& \left(u^{s}(x, t)-w_{\theta}^{s}(x, t)\right) \int_{\Omega} J(x-y)(u(y, t)-u(x, t)) d y \\
& =\left(u^{s}(x, t)-w_{\theta}^{s}(x, t)\right) \int_{\Omega^{+}} J(x-y)(u(y, t)-u(x, t)) d y \\
& \quad+\left(u^{s}(x, t)-w_{\theta}^{s}(x, t)\right) \int_{\Omega^{-}} J(x-y)(u(y, t)-u(x, t)) d y \leq 0 .
\end{aligned}
$$

In what follows, we use the ideas of [3].

Lemma 2.2 For any nonnegative and integrable functions $f, g$ and any $r \geq 1$, we have

$$
A_{u}(x, t) f g \leq\|f\|_{\infty}\left(A_{u}(x, t) g^{r}\right)^{1 / r} .
$$

Proof Let $u(x, t)=A_{u}(x, t) f(x) g(x)$ and $v(x, t)=\|f\|_{\infty}^{r}\left(A_{u}(x, t) g^{r}(x)\right)$. Using the mean value theorem and by Remark 2.2, we have

$$
\left(u^{r}(x, t)\right)_{t}-\int_{\Omega} J(x-y)\left(u^{r}(y, t)-u^{r}(x, t)\right) d y
$$




$$
\begin{aligned}
& =r u^{r-1}(x, t) u_{t}(x, t)-r \int_{\Omega} J(x-y)\left(w_{\theta}(x, t)\right)^{r-1}(u(y, t)-u(x, t)) d y \\
& =r\left(u^{r-1}(x, t)-w_{\theta}^{r-1}(x, t)\right) \int_{\Omega} J(x-y)(u(y, t)-u(x, t)) d y \leq 0
\end{aligned}
$$

with $w_{\theta}(x, t)=\theta u(y, t)+(1-\theta) u(x, t)$, for $\theta \in(0,1)$. Therefore $u^{r}(x, t)$ is a sub-solution of (1.4). Analogously, we have $v(x, t)$ is solution of (1.4). Moreover, $u^{r}(x, 0)=f^{r}(x) g^{r}(x) \leq$ $\|f\|_{\infty}^{r} g^{r}(x)=v(x, 0)$, then, by the comparison principle $u^{r}(x, t) \leq v(x, t)$ for all $(x, t) \in \Omega \times$ $(0, T)$. Therefore $A_{u}(x, t) f g \leq\|f\|_{\infty}\left(A_{u}(x, t) g^{r}\right)^{1 / r}$.

Now, we will study the existence and uniqueness of the solution for (1.6).

Theorem 2.1 If $p q \geq 1$ and $\left(u_{0}, v_{0}\right) \in C(\bar{\Omega}) \times C(\bar{\Omega})$ are nonnegative real functions then there exist a unique solution $(u, v)$ of $(1.6)$ such that $(u, v) \in P_{t_{0}}$.

Proof We shall proceed by considering various cases.

Case 1 . Let $p, q \geq 1$. Then the operator $\psi: P_{t_{0}} \rightarrow P_{t_{0}}$ is well defined. In fact, for any $(x, t) \in \bar{\Omega} \times\left[0, t_{0}\right]$ we have

$$
\begin{aligned}
& \left|T_{u_{0}}(u)(x, t)-u_{0}(x)\right| \\
& \quad \leq \int_{0}^{t} \int_{\Omega} J(x-y)|u(y, s)-u(x, s)| d y d s+\left.\left|\int_{0}^{t}\right| v\right|^{p-1} v(x, s) d s \mid \\
& \quad \leq K|\Omega| \int_{0}^{t}\|u(\cdot, s)\|_{L^{\infty}(\bar{\Omega})} d s+\int_{0}^{t}\|v(\cdot, s)\|_{L^{\infty}(\bar{\Omega})}^{p} d s \\
& \quad \leq \max \{1, K|\Omega|\} t\left(\|u\|_{\kappa}+\|v\|_{\kappa}^{p}\right) .
\end{aligned}
$$

Analogously we have

$$
\left|S_{\nu_{0}}(v)(x, t)-v_{0}(x)\right| \leq \max \{1, K|\Omega|\} t\left(\|v\|_{\kappa}+\|u\|_{\kappa}^{q}\right) .
$$

Therefore from (2.5) and (2.6) we conclude that $\psi$ is continuous at $t=0$. Now, for $\left(x, t_{1}\right),\left(x, t_{2}\right) \in \bar{\Omega} \times\left(0, t_{0}\right]$ with $t_{1}<t_{2}$, we have

$$
\begin{aligned}
& \left|T_{u_{0}}\left(u\left(x, t_{1}\right)\right)-T_{u_{0}}\left(u\left(x, t_{2}\right)\right)\right| \\
& \quad \leq \int_{t_{1}}^{t_{2}} \int_{\Omega} J(x-y)|u(y, s)-u(x, s)| d y d s+\left.\left|\int_{t_{1}}^{t_{2}}\right| v\right|^{p-1} v(x, s) d s \mid \\
& \quad \leq 2 k|\Omega| \int_{t_{1}}^{t_{2}}\|w(\cdot, s)\|_{L^{\infty}(\bar{\Omega})}+\int_{t_{1}}^{t_{2}}\|v(\cdot, s)\|_{L^{\infty}(\bar{\Omega})}^{p} d s \\
& \quad \leq 2 \max \{1, K|\Omega|\}\left(t_{2}-t_{1}\right)\left(\|u\|_{\kappa}+\|v\|_{\kappa}^{p}\right) .
\end{aligned}
$$

Analogously, we obtain

$$
\left|S_{v_{0}}\left(v\left(x, t_{2}\right)\right)-S_{v_{0}}\left(v\left(x, t_{1}\right)\right)\right| \leq 2 \max \{1, K|\Omega|\}\left(t_{2}-t_{1}\right)\left(\|v\|_{\kappa}+\|u\|_{\kappa}^{q}\right)
$$

Therefore using (2.7) and (2.8) we see that $\psi$ is continuous at $t \in\left(0, t_{0}\right]$. Since $J * u$ for $u \in C(\bar{\Omega})$ is also uniformly continuous, we have $\psi(u, v)$ is a continuous function of $x$. 
Therefore for any $\left(u_{0}, v_{0}\right) \in C(\bar{\Omega}) \times C(\bar{\Omega})$ and $(u, v) \in P_{t_{0}}$ we have $\psi(u, v) \in P_{t_{0}}$. Taking $\left(u_{0}, v_{0}\right)=\left(w_{0}, z_{0}\right)$ in Lemma 2.1 and choosing $t_{0}$ such that $C t_{0}<1$, ensures that $\psi(u, v)$ is a strict contraction in the ball $B=B\left(\left(u_{0}, v_{0}\right) ; 2\left\|\left(u_{0}, v_{0}\right)\right\|_{I}\right)$ in $P_{t_{0}}$. Indeed, for $(u, v),(w, z)$ in the ball we have $\mu, v \leq C\left\|\left(u_{0}, v_{0}\right)\right\|_{I}$ (with $v=\max \left\{\|u\|_{\kappa},\|w\|_{\kappa}\right\}, \mu=\max \left\{\|v\|_{\kappa},\|z\|_{\kappa}\right\}$ ), we obtain $\psi(u, v)$ is a strict contraction of $P_{t_{0}} \cap B$ into itself and therefore there exists a unique fixed point $(u, v)$ of $\psi(u, v)$ in $P_{t_{0}} \cap B$ according with the Banach fixed point theorem, which also give us the uniqueness of solution of $(1.6)$ in $\left[0, t_{0}\right]$. If $\|(u, v)\|<\infty$, taking as initial datum $\left(u\left(\cdot, t_{0}\right), v\left(\cdot, t_{0}\right)\right) \in C(\bar{\Omega}) \times C(\bar{\Omega})$ and arguing as before, it is possible to extend the solution up to some interval $\left[0, t_{1}\right)$, for certain $t_{1}>t_{0}$. Hence, we conclude the maximal existence time of the solution, $0<T \leq \infty$.

Case 2. Now, we consider $p \leq 1$ or $q \leq 1$. Following the ideas of the authors of [3] and taking by convenience $p<1$ and $q \geq 1$, we have $0<p<1<1 / p \leq q$. For $t_{0}>$ 0 , let $Y_{t_{0}}=\left\{u:\left[0, t_{0}\right] \rightarrow C(\bar{\Omega}): u\right.$ is continuous $\}$ the Banach space with norm $\|u\|_{\kappa}=$ $\max _{0 \leq t \leq t_{0}}\|u(\cdot, t)\|_{L^{\infty}(\bar{\Omega})}$ and $Y_{t_{0}}^{+}=\left\{u \in Y_{t_{0}}: u \geq 0\right\}$ a closed subspace of $Y_{t_{0}}$ and let $B_{R}=$ $\left\{u \in X_{t_{0}}:\|u\|_{\kappa}<R\right\}$ with $R>0$.

Recalling (2.1), we define the following operator $L_{u_{0}}: Y_{t_{0}}^{+} \rightarrow Y_{t_{0}}^{+}$,

$$
L_{u_{0}}(u)(x, t)=A_{u}(x, t) u_{0}+\int_{0}^{t}\left(A_{v}(x, s) v_{0}+\int_{0}^{s} u^{q}(x, \tau) d \tau\right)^{p} d s .
$$

We show that, for $R>0$ large enough and $t_{0}$ small enough, the mapping $L_{u_{0}}(u)(x, t)$ is a strict contraction from $Y_{t_{0}}^{+} \cap B_{R}$ into itself. Let $u, w \in Y_{t_{0}}^{+}$, using the mean value theorem we have

$$
\begin{aligned}
& \left|L_{u_{0}}(u(x, t))-L_{w_{0}}(w(x, t))\right| \\
& \leq \int_{0}^{t} \mid\left(A_{v}(x, s) v_{0}+\int_{0}^{s} u^{q}(x, \tau) d \tau\right)^{p} \\
& \quad-\left(A_{z}(x, s) z_{0}+\int_{0}^{s} w^{q}(x, \tau) d \tau\right)^{p} \mid d s \\
& \leq p \int_{0}^{t}\left(\int_{0}^{s}|\theta u(x, \tau)+(1-\theta) w(x, \tau)|^{q} d \tau\right)^{p-1} \\
& \quad \cdot\left(q \int_{0}^{s}|\theta u(x, \tau)+(1-\theta) w(x, \tau)|^{q-1}|u(x, \tau)-w(x, \tau)| d \tau\right) d s,
\end{aligned}
$$

for some $\theta=\theta(s) \in(0,1)$. Applying Lemma 2.2 in the above inequality, we have, for $u, w \in$ $Y_{t_{0}}^{+} \cap B_{R}$

$$
\left\|L_{u_{0}}(u)-L_{w_{0}}(w)\right\|_{\kappa} \leq p q R^{p q-1} C\left(t_{0}\right)\|u-w\|_{\kappa},
$$

where $C\left(t_{0}\right) \rightarrow 0$ as $t_{0} \rightarrow 0$.

Remark 2.3 We observe that the same argument as in the proof of Theorem 2.1 shows the existence of a unique solution of (1.6) if the reaction terms are replaced by $f(v), g(u)$ locally Lipschitz functions, respectively.

Theorem 2.2 Let $p q<1$ and $\left(u_{0}, v_{0}\right) \in C(\bar{\Omega}) \times C(\bar{\Omega})$ nonnegative functions, then there exists a solution $(u, v) \in P_{t_{0}}$ of $(1.6)$. 
Proof The existence of a solution for (1.6) is obtained by means of an approximation procedure as in [2]. We assume that $0<p<1 \leq q$, let $\left(f_{n}\right)_{n}$ be a sequence of globally Lipschitz functions such that, for $n$ fixed, $f_{n}(s)=0$, if $s \leq 0, f_{n}(s)=s^{p}$ if $s \geq \frac{1}{2 n}$, with $f_{n}$ nondecreasing and $\lim _{n \rightarrow \infty} f_{n}(s)=s^{p}$ for $s \geq 0$. Consider the problems

$$
\begin{aligned}
& u_{t}(x, t)=\int_{\Omega} J(x-y)(u(y, t)-u(x, t)) d y+f_{n}(v), \\
& v_{t}(x, t)=\int_{\Omega} J(x-y)(v(y, t)-v(x, t)) d y+|u|^{q-1}(x, t) u(x, t), \\
& u(x, 0)=u_{0}(x), \quad v_{n}(x, 0)=v_{0}(x)+\frac{1}{n}, \quad x \in \Omega .
\end{aligned}
$$

Arguing as Lemma 2.1 and Theorem 2.1, we see that (2.10) have a unique solution $\left(u_{n}(x, t), v_{n}(x, t)\right)$. As $v_{n}(0)>v_{m}(0)$ for $n<m$, we have $u_{m}(x, t)<u_{n}(x, t)$ and $v_{m}(x, t)<$ $v_{n}(x, t)$, therefore $\left(u_{n}(x, t)\right)_{n}$ and $\left(v_{n}(x, t)\right)_{n}$ are nondecreasing and bounded sequences. Therefore letting $n \rightarrow \infty$, there exists $(u(x, t), v(x, t))$, a solution of (1.6).

Now, as before, we obtain the following results.

Corollary 2.1 The solution $(u, v)$ of (1.6) depends continuously on the initial data. In fact if $(u, v)$ and $(w, z)$ are solutions of $(1.6)$ with initial data $\left(u_{0}, v_{0}\right)$ and $\left(w_{0}, z_{0}\right)$, respectively, then there exists a constant $\widetilde{C}=\widetilde{C}\left(t_{0}, K\right)$ such that

$$
\|(u, v)-(w, z)\| \leq \widetilde{C}\left\|\left(u_{0}, v_{0}\right)-\left(w_{0}, z_{0}\right)\right\|_{I} .
$$

Corollary 2.2 $(u(x, t), v(x, t)) \in P_{t_{0}}$ is a solution of $(1.6)$ :

$$
\begin{aligned}
& u(x, t)=\int_{0}^{t} \int_{\Omega} J(x-y)(u(y, s)-u(x, s)) d y d s+\int_{0}^{t} v^{p}(x, s) d s+u_{0}(x), \\
& v(x, t)=\int_{0}^{t} \int_{\Omega} J(x-y)(v(y, s)-v(x, s)) d y d s+\int_{0}^{t} u^{q}(x, s) d s+v_{0}(x) .
\end{aligned}
$$

Remark 2.4 Let us consider the following ODE system:

$$
\begin{array}{ll}
u^{\prime}(t)=v^{p}(t), \quad v^{\prime}(t)=u^{q}(t) \quad \text { for } t>0, \\
u(0)=a \geq 0, \quad v(0)=b \geq 0 .
\end{array}
$$

If $p q>1$, the solution of (2.12) is

$$
\begin{aligned}
u(t) & =C_{1}\left(\frac{(p q-1)^{(p+1) /(p q-1)}}{\left((p+1)(q+1)^{p}\right)^{1 /(p q-1)} a^{(p q-1) /(p+1)}}-t\right)^{-(p+1) /(p q-1)}, \\
C_{1} & =\left(\frac{(p+1)(q+1)^{p}}{(p q-1)^{(p+1)}}\right)^{1 /(p q-1)}, \\
v(t) & =C_{2}\left(\frac{(p q-1)^{(q+1) /(p q-1)}}{\left((p+1)^{q}(q+1)\right)^{1 /(p q-1)} b^{(p q-1) /(q+1)}}-t\right)^{-(q+1) /(p q-1)}, \\
C_{2} & =\left(\frac{(p+1)^{q}(q+1)}{(p q-1)^{(q+1)}}\right)^{1 /(p q-1)} .
\end{aligned}
$$


If $p q<1$, the solution of $(2.12)$ is

$$
\begin{array}{ll}
u(t)=\left(a^{(1-p q) /(p+1)}+C_{1} t\right)^{(p+1) /(1-p q)}, & C_{1}=\left(\frac{1-p q}{p+1}\right)\left(\frac{p+1}{q+1}\right)^{p /(p+1)}, \\
v(t)=\left(b^{(1-p q) /(q+1)}+C_{2} t\right)^{(q+1) /(1-p q)}, & C_{2}=\left(\frac{1-p q}{q+1}\right)\left(\frac{q+1}{p+1}\right)^{q /(q+1)} .
\end{array}
$$

If $p q=1$, the solution of $(2.12)$ is

$$
u(t)=a e^{C_{1} t}, \quad C_{1}=\left(\frac{p+1}{q+1}\right)^{p /(p+1)} ; \quad v(t)=b e^{C_{2} t}, \quad C_{2}=\left(\frac{q+1}{p+1}\right)^{q /(q+1)} .
$$

Remark 2.5 A flat solution of (1.6) is a solution that does not depend on $x$. $(u(t), v(t))$ is a flat solution of (1.6) with initial datum $u(x, 0)=u(0)=a, v(x, 0)=v(0)=b$ if and only if $(u(t), v(t))$ is a solution of $(2.12)$.

Remark 2.6 If $p q<1$, the solution of (1.6) is not unique. A solution of (1.6) with initial condition $\left(u_{0}, v_{0}\right)=(0,0)$ is given by $(u(x, t), v(x, t))=(0,0)$ for all $(x, t) \in \Omega \times(0, T)$. On the other hand, by $(2.14)$ we see that $u(t)=\left(C_{1} t\right)^{(p+1) /(1-p q)}, v(t)=\left(C_{2} t\right)^{(q+1) /(1-p q)}$ it is also a positive solution of (1.6) with initial condition $\left(u_{0}, v_{0}\right)=(0,0)$.

We will use the notation $(a, b) \geq(c, d)$ to indicate that $a \geq c$ and $b \geq d$.

Definition 2.1 Let $\bar{u}, \bar{v} \in C^{1}([0, T) ; C(\bar{\Omega})) .(\bar{u}, \bar{v})$ is called a super-solution of (1.6) if

$$
\begin{aligned}
& \bar{u}_{t}(x, t) \geq \int_{\Omega} J(x-y)(\bar{u}(y, t)-\bar{u}(x, t)) d y+\bar{v}^{p}(x, t), \\
& \bar{v}_{t}(x, t) \geq \int_{\Omega} J(x-y)(\bar{v}(y, t)-\bar{v}(x, t)) d y+\bar{u}^{q}(x, t), \\
& \bar{u}(x, 0) \geq u_{0}(x), \quad \bar{v}(x, 0) \geq v_{0}(x), \quad x \in \Omega .
\end{aligned}
$$

Analogously $(\underline{u}, \underline{v}) \in P_{t_{0}}$ is called a sub-solution of (1.6) if it satisfies the opposite inequalities.

Lemma 2.3 (Comparison principle) Let $(\underline{u}, \underline{v})$ and $(\bar{u}, \bar{v})$ be a sub-solution and supersolution of (1.6), respectively. If $\left(\underline{u}_{0}(x), \underline{v}_{0}(x)\right) \leq\left(\bar{u}_{0}(x), \bar{v}_{0}(x)\right)$ for all $x \in \Omega$, then $(\underline{u}(x, t)$, $\underline{v}(x, t)) \leq(\bar{u}(x, t), \bar{v}(x, t))$ for all $(x, t) \in \Omega \times(0, T)$.

Proof Let $w(x, t)=\bar{u}(x, t)-\underline{u}(x, t), z(x, t)=\bar{v}(x, t)-\underline{v}(x, t)$. Assume first that $w(x, 0), z(x, 0)>$ 0 for $x \in \Omega$. We observe that $w$ and $z$ verify

$$
\begin{aligned}
& w_{t}(x, t) \geq \int_{\Omega} J(x-y)(w(y, t)-w(x, t)) d y+\left(\frac{\bar{\nu}^{p}(x, t)-\underline{v}^{p}(x, t)}{\bar{v}(x, t)-\underline{v}(x, t)}\right) z(x, t), \\
& z_{t}(x, t) \geq \int_{\Omega} J(x-y)(z(y, t)-z(x, t)) d y+\left(\frac{\bar{u}^{q}(x, t)-\underline{u}^{q}(x, t)}{\bar{u}(x, t)-\underline{u}(x, t)}\right) w(x, t) .
\end{aligned}
$$

Now, set $0<\delta=\min \{w(x, 0), z(x, 0)\}$ and suppose that the conclusion of the lemma is false. Thus, let $t_{1}$ be the first time that $\delta / 2=\min \left\{w\left(x, t_{1}\right), z\left(x, t_{1}\right)\right\}$. We can assume that $w$ attains 
the minimum. In that time, there exists $x_{1} \in \Omega$ such that $w\left(x_{1}, t_{1}\right)=\delta / 2$. However, we have $w_{t}\left(x_{1}, t_{1}\right) \leq 0$ and, on the other hand,

$$
\begin{aligned}
w_{t}\left(x_{1}, t_{1}\right) & \geq \int_{\Omega} J\left(x_{1}-y\right)\left(w(y, t)-w\left(x_{1}, t_{1}\right)\right) d y+\left(\frac{\bar{\nu}^{p}\left(x_{1}, t_{1}\right)-\underline{v}^{p}\left(x_{1}, t_{1}\right)}{\bar{v}\left(x_{1}, t_{1}\right)-\underline{v}\left(x_{1}, t_{1}\right)}\right) z\left(x_{1}, t_{1}\right) \\
& \geq p \eta^{p-1}\left(x_{1}, t_{1}\right) z\left(x_{1}, t_{1}\right)>0
\end{aligned}
$$

where $\underline{v}\left(x_{1}, t_{1}\right)<\eta\left(x_{1}, t_{1}\right)<\bar{v}\left(x_{1}, t_{1}\right)$, which is a contradiction. Using the continuity of solutions of (1.6) with respect to the initial condition and an approximation argument, the result follows for the general initial condition.

Corollary 2.3 Let $(\bar{u}, \bar{v}) \in P_{t_{0}}$ a super-solution of (1.6). Them, if $\left(u_{0}, v_{0}\right) \geq(0,0)$ for $x \in \Omega$ we have $(\bar{u}(x, t), \bar{v}(x, t)) \geq(0,0)$ for all $(x, t) \in \Omega \times(0, T)$ and, moreover, a strict inequality holds if $\left(u_{0}, v_{0}\right)$ is positive.

Remark 2.7 Consider the problem (1.6) with initial datum $\left(u_{0}, v_{0}\right)$. Let $p q<1$, by Remark 2.4, we see that $(\bar{u}(t), \bar{v}(t))$ is the solution of (2.12) with $a \geq u_{0}, b \geq v_{0}$. Then, $(\bar{u}(t), \bar{v}(t))$ is a super-solution of (1.6). If $(u(x, t), v(x, t))$ is a solution of (1.6) with initial conditions $\left(u_{0}(x), v_{0}(x)\right)$, then by the comparison principle Lemma 2.3 we have $u(x, t) \leq \bar{u}(t)$ and $v(x, t) \leq \bar{v}(t)$ in $\Omega \times(0, T)$. Therefore any solution of (1.6) can be continued for al times in the case $p q<1$.

The following lemma confirms the existence of a maximal solution of (1.6) if $p q<1$. Its proof is analogous to those given in Lemma 2.4 in [3]; therefore we omit here.

Lemma 2.4 Let $0<p q<1$. Then there exists a maximal solution $\left(u_{M}(x, t), v_{M}(x, t)\right)$ of $(1.6)$ in the sense that if $(u(x, t), v(x, t))$ is any other solution we have

$$
u(x, t) \leq u_{M}(x, t), \quad v(x, t) \leq v_{M}(x, t) \quad \text { in } \Omega \times(0, \infty) .
$$

Moreover, $u_{M}(x, t)>0, v_{M}(x, t)>0$ for all $(x, t) \in \Omega \times(0, \infty)$.

Theorem 2.3 Let $\left(u_{0}, v_{0}\right)=(0,0)$.

1. If $p q \geq 1$, then the unique solution of $(1.6)$ is $(u, v)=(0,0)$.

2. If $p q<1$, then there exists exactly one solution $(u, v)$ of $(1.6)$ such that $u$ and $v$ are positive.

Proof 1. If $p q \geq 1$, the uniqueness of solution of (1.6) is given in Theorem 2.1.

2. Let $p q<1$ and $(\mu(t), v(t))$ be the positive solution given in Remark 2.6. Let $0<$ $a, b<1$ be constants such that $(\underline{u}(x, t), \underline{v}(x, t))=(a \mu(t), b v(t))$ is a sub-solution of $(1.6)$ in $\Omega \times\left(0, t_{0}\right)$. Let $n \in \mathbb{N}$ be given and consider locally Lipschitz functions $f_{n}, g_{n}$ such that $f_{n}(s)=s^{p}, g_{n}(s)=s^{q}$ for $s \geq 1 / 2 n$. It follows from Remark 2.3 there exists a unique solution $\left(u_{n}(x, t), v_{n}(x, t)\right)$ of (1.6) with the reaction terms replaced by $f_{n}(s), g_{n}(s)$ and initial conditions $u_{n}(x, 0)=v_{n}(x, 0)=1 / n$. By the comparison principle Lemma 2.3, we have $\left(u_{n}(x, t), v_{n}(x, t)\right) \geq(\underline{u}(x, t), \underline{v}(x, t))$ and the sequences $\left(u_{n}\right),\left(v_{n}\right)$ are monotone decreasing in $n$. We have $\left(u_{n}, v_{n}\right)$ for every $n$ is defined on the interval $\left[0, t_{1}\right]$ where $\left(u_{1}, v_{1}\right)$ are defined. Then, by the monotone convergence theorem, we obtain $\lim _{n \rightarrow \infty} u_{n}=u, \lim _{n \rightarrow \infty} v_{n}=v$, $u(x, 0)=0, v(x, 0)=0$. By Corollary 2.2, $(u, v)$ is a positive solution of $(1.6)$ with initial conditions $\left(u_{0}, v_{0}\right)=(0,0)$. 
In remains to prove the statement about uniqueness. Suppose for contradiction, there exists another positive solution $(w, z)$ of $(1.6)$ with initial conditions $\left(u_{0}, v_{0}\right)=(0,0)$. Using the comparison principle $u(x, t) \leq w(x, t+\tau), v(x, t) \leq z(x, t+\tau)$ for all $\tau>0$. Letting $\tau \rightarrow 0$, we see that $u(x, t) \leq w(x, t), v(x, t) \leq z(x, t)$. Analogously, we have $u(x, t) \geq w(x, t), v(x, t) \geq$ $z(x, t)$, therefore $u(x, t)=w(x, t), v(x, t)=z(x, t)$ for all $(x, t) \in \Omega \times(0, \infty)$.

Theorem 2.4 If $\left(u_{0}, v_{0}\right) \neq(0,0)$, then the solution of problem (1.6) is unique.

Proof We shall proceed by considering various cases.

Case 1. If $p q \geq 1$, its conclusion is obtained from Theorem 2.1.

Case 2. Let $p q<1$ and $p<1, q \leq 1$. We argue again by contradiction, and assume that (1.6) has two different solutions with $\left(u_{0}, v_{0}\right) \neq(0,0)$ initials conditions. Namely $\left(u_{M}(x, t), v_{M}(x, t)\right)$ the maximal solution constructed in Lemma 2.4 and $(\widetilde{u}(x, t), \widetilde{v}(x, t))$. We have $(\widetilde{u}(x, t), \widetilde{v}(x, t)) \leq\left(u_{M}(x, t), v_{M}(x, t)\right)$ in $\Omega \times[0, \infty)$. We prove $(\widetilde{u}(x, t), \widetilde{v}(x, t)) \geq$ $\left(u_{M}(x, t), v_{M}(x, t)\right)$ in $\Omega \times[0, \infty)$. To this end, let $w(x, t)=u_{M}(x, t)-\widetilde{u}(x, t), z(x, t)=v_{M}(x, t)-$ $\widetilde{v}(x, t)$, we have

$$
\begin{aligned}
w_{t}(x, t) & =\int_{\Omega} J(x-y)(w(y, t)-w(x, t)) d y+v_{M}^{p}(x, t)-\widetilde{v}^{p}(x, t) \\
& \leq \int_{\Omega} J(x-y)(w(y, t)-w(x, t)) d y+z^{p}(x, t), \\
z_{t}(x, t) & \leq \int_{\Omega} J(x-y)(z(y, t)-z(x, t)) d y+w^{q}(x, t), \\
w(x, 0) & =z(x, 0)=0, \quad x \in \Omega .
\end{aligned}
$$

Therefore $(w(x, t), z(x, t))$ is a sub-solution of (1.6) with $\left(w_{0}(x), z_{0}(x)\right) \leq\left(u_{0}(x), v_{0}(x)\right)$ for $x \in \Omega$. Them by Lemma 2.3 , we have $(w(x, t), z(x, t)) \leq(u(x, t), v(x, t))$ in $\Omega \times[0, \infty)$, where $(u(x, t), v(x, t))$ is the unique solution, positive, of (1.6).

Let $f(x, t)=u(x, t)-w(x, t) \geq 0, g(x, t)=v(x, t)-z(x, t) \geq 0$. As $p<1, q \leq 1, v, v_{M}, \widetilde{v}$, $u, u_{M}, \widetilde{u}$ are nonnegative and $u_{M} \geq \widetilde{u}, v_{M} \geq \widetilde{v}$, then $\left(v^{p}-v_{M}^{p}+\widetilde{v}^{p}\right) \geq\left(v-v_{M}+\widetilde{v}\right)^{p}$ and $\left(u^{q}-u_{M}^{q}+\widetilde{u}^{q}\right) \geq\left(u-u_{M}+\widetilde{u}\right)^{q}$. Therefore

$$
\begin{aligned}
& f_{t}(x, t) \geq \int_{\Omega} J(x-y)(f(y, t)-f(x, t)) d y+g^{p}(x, t), \\
& g_{t}(x, t) \geq \int_{\Omega} J(x-y)(g(y, t)-g(x, t)) d y+f^{q}(x, t),
\end{aligned}
$$

with $f(x, 0)=g(x, 0)=0, x \in \Omega$. We claim that $f(x, t)>0, g(x, t)>0$ for all $(x, t) \in \Omega \times(0, \infty)$. Indeed if not, suppose there exists $0<t_{0}$ such that $f(x, t)=g(x, t)=0$ for all $(x, t) \in \Omega \times$ $\left(0, t_{0}\right]$. We have

$$
\begin{aligned}
(v(x, t)+\widetilde{v}(x, t))^{p} & \\
= & \left.v_{M}^{p} x, t\right)=\left(u_{M}\right)_{t}(x, t)-\int_{\Omega} J(x-y)\left(u_{M}(y, t)-u_{M}(x, t)\right) d y \\
= & \left(u_{t}(x, y)+\widetilde{u}_{t}(x, t)\right) \\
& \quad-\left(\int_{\Omega} J(x-y)(u(y, t)-u(x, t)) d y-\int_{\Omega} J(x-y)(\widetilde{u}(y, t)-\widetilde{u}(x, t)) d y\right) \\
= & v^{p}(x, t)+\widetilde{v}^{p}(x, t) .
\end{aligned}
$$


Therefore $(v(x, t)+\widetilde{v}(x, t))^{p}=v^{p}(x, t)+\widetilde{v}^{p}(x, t)$ for all $(x, t) \in \Omega \times\left(0, t_{0}\right]$. Analogously, we have $(u(x, t)+\widetilde{u}(x, t))^{q}=u^{q}(x, t)+\widetilde{u}^{q}(x, t)$ for all $(x, t) \in \Omega \times\left(0, t_{0}\right]$. This is a contradiction since $u(x, t)>0, v(x, t)>0$ for all $(x, t) \in \Omega \times(0, \infty)$ and $p<1$. The claim is proved.

By the comparison principle Lemma 2.3, we have $f(x, t) \leq u(x, t), g(x, t) \leq u(x, t)$ in $\Omega \times$ $(0, \infty)$. This implies $(\widetilde{u}, \widetilde{v}) \geq\left(u_{M}, v_{M}\right)$ in $\Omega \times[0, \infty)$.

Case 3. Let $p q<1$, with $0<p<1<q$. We reduce this case to Case 2. Let $w(x, t)=$ $\mu\left(u_{M}(x, t)-\widetilde{u}(x, t)\right), z(x, t)=v\left(v_{M}(x, t)-\widetilde{v}(x, t)\right)$ in $\Omega \times[0, \infty)$, with $\mu, v$ constant to be determined. Since $p<1$, we have

$$
\begin{aligned}
& w_{t}(x, t)-\int_{\Omega} J(x-y)(w(y, t)-w(x, t)) d y=\mu\left(v_{M}^{p}(x, t)-\widetilde{v}^{p}(x, t)\right) \leq v \mu^{-p} z(x, t), \\
& z_{t}(x, t)-\int_{\Omega} J(x-y)(z(y, t)-z(x, t)) d y=v\left(u_{M}^{q}(x, t)-\widetilde{u}^{q}(x, t)\right), \\
& w(x, 0)=z(x, 0)=0, \quad x \in \Omega .
\end{aligned}
$$

As $q>1$, we have

$$
u_{M}^{q}(x, t)-\widetilde{u}^{q}(x, t)=\frac{u_{M}^{q}(x, t)-\widetilde{u}^{q}(x, t)}{u_{M}(x, t)-\widetilde{u}(x, t)}\left(u_{M}(x, t)-\widetilde{u}(x, t)\right) \leq \mu^{-1} M w(x, t)
$$

for some constant $M>0$ that depends on the bound for $u_{M}$ on $\Omega \times[0, \infty)$ and of $q$. Therefore, $\mu, v$ can be chosen to be positive and such that $(w, z)$ satisfies $w(x, t) \leq v \mu^{-p} z(x, t)$, $z(x, t) \leq v \mu^{-1} M w(x, t), w(x, 0)=z(x, 0)$ in $\Omega$. Now, we argue as in Case 2 , and we find $(\widetilde{u}, \widetilde{v}) \geq\left(u_{M}, v_{M}\right)$ in $\Omega \times[0, \infty)$.

\section{Global existence and blow-up}

In this section, we study the conditions under which the solutions of Eq. (1.6) blow up in finite time or are global.

Theorem 3.1 Let $p q>1$ and $u_{0}, v_{0} \in C(\bar{\Omega})$ nonnegative and nontrivial functions. The solution $(u, v)$ of $(1.6)$ blows up in finite time $T$.

Proof Let $p q>1$ and $u_{0}(x), v_{0}(x) \in C(\bar{\Omega})$ be nonnegative and nontrivial functions and $(u, v)$ solution of (1.6). We shall proceed by considering various cases.

Case 1. Suppose there exists a constant $c>0$ such that $u_{0} \geq c>0$ and $v_{0} \geq c>0$ for all $x \in \Omega$. Let $(w(t), z(t))$ a solution of (2.12) with $w(0)=z(0)=c$ initial condition. Moreover, we have $(w(t), z(t))$ is solution of (1.6), then by the comparison principle Lemma 2.3 we have $(w(t), z(t)) \leq(u(x, t), v(x, t))$. As $p q>1$ and by (2.13) we have $(w(t), z(t))$ blows up in finite time $\widetilde{T}>0$, then $(u, v)$ blows up in finite time $T<\widetilde{T}$.

Case 2. Suppose that $u_{0} \neq 0$. As the solutions $(u, v)$ of $(1.6)$ are nonnegative, by (1.6) we have

$$
u_{t}(x, t) \geq \int_{\Omega} J(x-y)(u(y, t)-u(x, t)) d y .
$$

Consider the problem

$$
\begin{aligned}
& \underline{u}_{t}(x, t)=\int_{\Omega} J(x-y)(\underline{u}(y, t)-\underline{u}(x, t)) d y, \quad x \in \Omega, t>0, \\
& \underline{u}(x, 0)=u_{0}(x), \quad x \in \Omega .
\end{aligned}
$$


From (3.1) we see that $u$ is a super-solution of (3.2), by the comparison principle (see [18]) we have $\underline{u}(x, t) \leq u(x, t)$.

Now, we assert that, for $\delta>0$, we obtain $\underline{u}(x, \delta) \geq c>0$ in $\Omega$. In fact, otherwise, there exists $x_{0} \in \Omega$ such that $\underline{u}\left(x_{0}, \delta\right)=0$ and

$$
0 \geq \underline{u}_{t}\left(x_{0}, \delta\right)=\int_{\Omega} J\left(x_{0}-y\right)\left(\underline{u}(y, \delta)-\underline{u}\left(x_{0}, \delta\right)\right) d y \geq 0 .
$$

As $J$ is supported in the unitary ball, then $\int_{\Omega} J\left(x_{0}-y\right)(\underline{u}(y, \delta)) d y=0$ in $B\left(x_{0}, 1\right)$ (unit ball with center in $\left.x_{0}\right)$. Repeating the above analysis in any $x \in B\left(x_{0}, 1\right)$, we obtain $\int_{\Omega} J\left(x_{0}-\right.$ $y)(\underline{u}(y, \delta)) d y=0$ for all $x_{0} \in \Omega$, since $\Omega$ is connected, therefore $\int_{\Omega} \underline{u}(y, \delta) d y=0$ for all $x_{0} \in \Omega$. By the conservation of mass (see [18]), we have

$$
0=\int_{\Omega} \underline{u}(x, \delta) d x=\int_{\Omega} \underline{u}(x, 0) d x=\int_{\Omega} u_{0}(x) d x>0
$$

which is a contradiction.

Therefore, for $\delta>0$, we obtain $u(x, \delta) \geq c>0$ and $v(x, \delta) \geq c>0$, which leads to Case 1 , therefore $(u, v)$ blows up in finite time. Let us see that $v(x, \delta) \geq c>0$. Suppose that $v_{0}=0$ in $\Omega$ and for $\delta>0$, let $\delta / 2 \leq t$ with $u(x, t) \geq c>0$. From (1.6), we have

$$
v_{t}(x, t) \geq \int_{\Omega} J(x-y)(v(y, t)-u(x, t)) d y+c^{q} .
$$

Consider the problem

$$
\begin{aligned}
& \underline{v}_{t}(x, t)=\int_{\Omega} J(x-y)(\underline{v}(y, t)-\underline{v}(x, t)) d y, \quad x \in \Omega, t>\delta / 2, \\
& \underline{v}(x, \delta / 2)=0, \quad x \in \Omega .
\end{aligned}
$$

Therefore $\underline{v}(x, t)=c^{q} \delta / 2$ for $t>\delta / 2$. Moreover, from (3.4), $v(x, t)$ is a super-solution of (3.5) then by the comparison principle (see [18]) we have $\underline{v}(x, t) \leq v(x, t)$, then $v(x, t) \geq c>0$.

Theorem 3.2 Let $p q \leq 1$ and $u_{0}(x), v_{0}(x) \in C(\bar{\Omega})$ be nonnegative and nontrivial functions. Then the solution $(u, v)$ of $(1.6)$ exists globally.

Proof Let $p q \leq 1$ and $u_{0}(x), v_{0}(x) \in C(\bar{\Omega})$ nonnegative and nontrivial functions and $(u, v)$ solution of (1.6). We shall proceed by considering various cases.

Case 1. Let assume $p q<1$. Let $(w(t), z(t))$ the solution of (2.12) with $(w(0), z(0))$ initial condition such that $u_{0} \leq w(0), v_{0} \leq z(0)$. As $p q<1$, by (2.14), we see that $(w(t), z(t))$ exists for all $t>0$. Moreover, $(w(t), z(t))$ is a super-solution of (1.6), then $u(x, t) \leq w(t)$ and $v(x, t) \leq z(t)$ for all $(x, t) \in \Omega \times[0, \infty)$.

Case 2. Suppose now $p q=1$ and $q \geq 1$. We see that, for all $a>0,(\bar{u}(x, t), \bar{v}(x, t))=$ $\left(a e^{t}, a^{q} e^{q t}\right)$ is a super-solution of (1.6). Indeed, replacing in (1.6) we have $a e^{t} \geq a^{p q} e^{p q t}$, $q a^{q} e^{q t} \geq a^{q} e^{q t}$ if $p q=1$ and $q \geq 1$. Is suffices to choose $a \geq \max \left(\left\|u_{0}\right\|_{\infty},\left\|v_{0}\right\|_{\infty}^{1 / q}\right)$.

Next, we study the blow-up rate of the solutions of (1.6). We assume that $x=0 \in \Omega$. 
Theorem 3.3 Let $p q>1$ and $u_{0}(x), v_{0}(x) \in C(\bar{\Omega})$ be nonnegative and nontrivial functions. Let $(u, v)$ be the solution of $(1.6)$ such that the maximum is reached at $x=0$. Them exist $C_{1}$, $C_{2}, C_{3}, C_{4}$, positive constants such that

$$
\begin{aligned}
& C_{1}(T-t)^{-(p+1) /(p q-1)} \leq u(0, t) \leq C_{2}(T-t)^{-(p+1) /(p q-1)}, \\
& C_{3}(T-t)^{-(q+1) /(p q-1)} \leq v(0, t) \leq C_{4}(T-t)^{-(q+1) /(p q-1)} .
\end{aligned}
$$

Proof As $p q>1$, we see that $(u, v)$, the solution of (1.6) blows up in finite time $T$. Let $u(0, t)=\max _{x \in \bar{\Omega}} u(x, t)$ and $v(0, t)=\max _{x \in \bar{\Omega}} v(x, t)$. Due to (1.6), we have

$$
\begin{aligned}
& u_{t}(0, t)=\int_{\Omega} J(0-y)(u(y, t)-u(0, t)) d y+v^{p}(0, t) \leq v^{p}(0, t), \\
& v_{t}(0, t)=\int_{\Omega} J(0-y)(v(y, t)-v(0, t)) d y+u^{q}(0, t) \leq u^{q}(0, t) .
\end{aligned}
$$

As $1=\int_{\mathbb{R}^{N}} J(\zeta) d \zeta \geq \int_{\Omega} J(\zeta) d \zeta$, we have

$$
\begin{aligned}
u_{t}(0, t) & =\int_{\Omega} J(0-y)(u(y, t)-u(0, t)) d y+v^{p}(0, t) \\
& \geq-u(0, t)+v^{p}(0, t), \\
v_{t}(0, t) & =\int_{\Omega} J(0-y)(v(y, t)-v(0, t)) d y+u^{q}(0, t) \\
& \geq-v(0, t)+u^{q}(0, t) .
\end{aligned}
$$

Therefore, we have for all $0<t<T$

$$
-u(0, t)+v^{p}(0, t) \leq u_{t}(0, t) \leq v^{p}(0, t)
$$

and

$$
-v(0, t)+u^{q}(0, t) \leq v_{t}(0, t) \leq u^{q}(0, t) .
$$

Multiplying the second inequality of (3.9) with $u^{q}(0, t)$ and the first inequality of (3.10) with $v^{p}(0, t)$, we have

$$
u_{t}(0, t) u^{q}(0, t) \leq v_{t}(0, t) v^{p}(0, t)+v^{p+1}(0, t)
$$

which is equivalent to

$$
\left(\frac{u^{q+1}(0, t)}{q+1}\right)_{t} \leq\left(\frac{\nu^{p+1}(0, t)}{p+1}\right)_{t}+\nu^{p+1}(0, t)
$$

Multiplying the inequality with $(p+1) e^{(p+1) t}$ and integrating on $[0, t], t<T$, we have

$$
\begin{aligned}
u^{q}(0, t) & \leq\left((q+1) e^{(p+1) t} v^{p+1}(0, t)+C\right)^{q /(q+1)} \\
& \leq\left((q+1) e^{(p+1) T} v^{p+1}(0, t)+C\right)^{q /(q+1)} \\
& \leq C(v(0, t))^{(p+1) q /(q+1)} .
\end{aligned}
$$


Replacing the second inequality of (3.10) by the inequality (3.11), we have

$$
v_{t}(0, t) \leq C(v(0, t))^{(p+1) q /(q+1)} .
$$

Integrating the inequality from above on $[t, T)$, we obtain

$$
v(0, t) \geq C_{3}(T-t)^{-\beta},
$$

where $\beta=\frac{q+1}{p q-1}$.

In analogous form we obtain

$$
u(0, t) \geq C_{1}(T-t)^{-\alpha},
$$

where $\alpha=\frac{p+1}{p q-1}$.

Doing a similar analysis to the one developed above, we see that there exists a constant $C>0$ such that for $0<t<T$

$$
C(v(0, t))^{(p+1) q /(q+1)} \leq u^{q}(0, t) .
$$

Replacing the first inequality of (3.10) with the inequality (3.12) and as $p q>1$ we have $(p+1) q /(q+1)>1$ and

$$
C(v(0, t))^{(p+1) q /(q+1)} \leq-v(0, t)+C(v(0, t))^{(p+1) q /(q+1)} \leq v_{t}(0, t) .
$$

Integrating the inequality from above on $[t, T)$, we obtain

$$
v(0, t) \leq C_{4}(T-t)^{-\beta}
$$

Analogously we obtain

$$
u(0, t) \leq C_{2}(T-t)^{-\alpha} .
$$

Remark 3.1 If $p q>1$, by Remark 2.4, we see that the flat solution $(u(t), v(t))$ of (1.6) blows up in the whole $\bar{\Omega}$, in finite time $T$ for any non-negative initial condition.

\section{Conclusions}

In this paper, we have studied the existence and uniqueness of nonnegative solutions $(u, v)$ for (1.6). We have showed the solution $(u, v)$ is unique if $p q \geq 1$ or if one of the initial conditions is not zero for $p q<1$. The global existence for the solution of (1.6) has been analyzed. We have showed that if $p q>1$ and $u_{0}, v_{0}$ are nonnegative and nontrivial functions, the solution $(u, v)$ of $(1.6)$ blows up in finite time $T$, if $p q \leq 1$ the solution $(u, v)$ exists globally. Finally, we studied the blow-up rates for the solution $(u, v)$ of $(1.6)$. The results obtained allow us to conclude the system (1.6) shares many important properties with the corresponding local diffusion coupled parabolic system with Neumann boundary conditions (1.1). 
Competing interests

The author declares to have no competing interests.

\section{Authors' contributions}

The author contributed equally and significantly in writing this article. The author read and approved the final manuscript.

\section{Author's information}

Mauricio Bogoya is professor of Mathematic Department of Universidad Nacional de Colombia, Bogotá, Colombia.

\section{Publisher's Note}

Springer Nature remains neutral with regard to jurisdictional claims in published maps and institutional affiliations.

Received: 9 August 2017 Accepted: 13 March 2018 Published online: 27 March 2018

\section{References}

1. Deng, K.: Blow-up rates for parabolic systems. Z. Angew. Math. Phys. 47, 132-143 (1996)

2. Escobedo, M., Herrero, M.A.: Boundedness and blow up for a semilinear reaction diffusion system. J. Differ. Equ. 89, 176-202 (1981)

3. Escobedo, M., Herrero, M.A.: A semilinear parabolic system in a bounded domain. Ann. Mat. Pura Appl. (4) 165, 315-336 (1993)

4. Friedman, A., Giga, Y.: A single point blow-up for solutions of semi-linear parabolic systems. J. Fac. Sci., Univ. Tokyo, Sect. 1A, Math. 34, 65-79 (1987)

5. Pao, C.V.: Nonlinear Parabolic an Elliptic Equations. Plenum, New York (1992)

6. Pao, C.V.: On nonlinear reaction-diffusion systems. J. Math. Anal. Appl. 87, 165-198 (1982)

7. Quittner, P., Souplet, P.: Superlinear Parabolic Problems. Blow-up, Global Existence and Steady States. Birkhäuser, Basel (2007)

8. Wang, M.: Blow-up rate estimates for semilinear parabolic systems. J. Differ. Equ. 170, 317-324 (2001)

9. Friedman, A., McLeod, B.: Blow-up of positive solutions of semilinear heat equations. Indiana Univ. Math. J. 34, 425-447 (1985)

10. Friedman, A.: Blow-up of solutions of nonlinear parabolic equations. In: Ni, W.-M., Peletier, L.A., Serrin, J. (eds.) Nonlinear Diffusion Equations and Their Equilibrium States, vol. 1, pp. 301-318. Birkhäuser, Basel (1988)

11. Mueller, C.E., Weissler, F.B.: Single point blow-up for a general semilinear heat equation. Indiana Univ. Math. J. 34, $881-913$ (1983)

12. Samarskii, A.A., Galaktionov, V.A., Kurdyumov, S.P., Mikhailov, A.P.: Blow-up in Quasilinear Parabolic Equations. de Gruyter, Berlin (1995)

13. Shahmurov, R.: Solution of the Dirichlet and Neumann problems for a modified Helmholtz equation in Besov spaces on an annulus. J. Differ. Equ. 249(3), 526-550 (2010)

14. Bates, P., Fife, P., Ren, X., Wang, X.: Travelling waves in a convolution model for phase transitions. Arch. Ration. Mech. Anal. 138, 105-136 (1997)

15. Bogoya, M.: A nonlocal nonlinear diffusion equation in higher space dimensions. J. Math. Anal. Appl. 344, 601-615 (2008)

16. Cortázar, C., Elgueta, M., Rossi, J.D.: A non-local diffusion equation whose solutions develop a free boundary. Ann. Henri Poincaré 6(2), 269-281 (2005)

17. Fife, P.: Some nonclassical trends in parabolic and parabolic-like evolutions. In: Trends in Nonlinear Analysis, pp. 153-191. Springer, Berlin (2003)

18. Chasseigne, E., Chaves, M., Rossi, J.D.: Asymptotic behaviour for nonlocal diffusion equations. J. Math. Pures Appl. 86 , 271-291 (2006)

19. Pérez Llanos, M., Rossi, J.D.: Blow-up for a non-local diffusion problem with Neumann boundary conditions and a reaction term. Nonlinear Anal. TMA 70(4), 1629-1640 (2009)

20. García-Melián, J., Quirós, F.: Fujita exponents for evolution problems with nonlocal diffusion. J. Evol. Equ. 10, 147-161 (2010)

21. Amann, H.: Nonlocal quasilinear parabolic equations. Russ. Math. Surv. 60(6), 1021-1033 (2005)

\section{Submit your manuscript to a SpringerOpen ${ }^{\circ}$ journal and benefit from:}

- Convenient online submission

- Rigorous peer review

- Open access: articles freely available online

- High visibility within the field

- Retaining the copyright to your article

Submit your next manuscript at $\boldsymbol{\nabla}$ springeropen.com 